Materials Sciences Division, Lawrence Berkeley National Laboratory, and Department of Materials Science and Engineering University of California at Berkeley

\title{
MECHANISTIC FRACTURE CRITERIA FOR THE FAILURE OF HUMAN CORTICAL BONE
}

\author{
R. K. Nalla ${ }^{1}$, J. H. Kinney ${ }^{2}$ and R. O. Ritchie ${ }^{1,3}$ \\ ${ }^{1}$ Materials Sciences Division, Lawrence Berkeley National Laboratory, and \\ Department of Materials Science and Engineering, \\ University of California, Berkeley, CA 94720 \\ ${ }^{2}$ Department of Preventive and Restorative Dental Sciences, \\ University of California, San Francisco, CA 94143 \\ ${ }^{3}$ Corresponding author: Tel: (510) 486-5798; Fax: (510) 486-4881 \\ E-mail address: RORitchie@lbl.gov (R. O. Ritchie)
}

December 2002

submitted to the Nature Materials

This work was supported in part by the National Institutes of Health, National Institute for Dental and Craniofacial Research under Grant No. P01DE09859 and by the Office of Science, Office of Basic Energy Science of the Department of Energy under Contract No. DE-Ac03-76SF00098 


\title{
Mechanistic Fracture Criteria for the Failure of Human Cortical Bone
}

\author{
R. K. Nalla ${ }^{1}$, J. H. Kinney ${ }^{2}$ and R. O. Ritchie ${ }^{1}$ \\ ${ }^{1}$ Materials Sciences Division, Lawrence Berkeley National Laboratory, and Department \\ of Materials Science and Engineering, University of California, Berkeley, California \\ 94720, USA \\ ${ }^{2}$ Department of Preventive and Restorative Dental Sciences, University of California, \\ San Francisco, California 94143, USA.
}

Correspondence should be addressed to R.O.R. (Tel: (510) 486-5798; Fax: (510) 486-4881; e-mail: RORitchie@lbl.gov).

\begin{abstract}
A mechanistic understanding of fracture in human bone is critical to predicting fracture risk associated with age and disease. Despite extensive work, a mechanistic framework for describing how the underlying microstructure affects the failure mode in bone is lacking. Though micromechanical models incorporating local failure criteria have been widely developed for metallic and ceramic materials ${ }^{1,2}$, few such models exist for biological materials. In fact, there is no proof to support the widely held belief that fracture in bone is locally straincontrolled $^{3,4}$, as for example has been shown for ductile fracture in metallic materials ${ }^{5}$. In the present study, we provide such evidence through a novel series of experiments involving a double-notch-bend test geometry designed to shed light on the nature of the critical failure events in bone. We examine how the propagating crack interacts with the microstructure of human bone to provide some mechanistic understanding of fracture and to define how the properties vary with orientation. It was found that fracture in human cortical bone is consistent with strain-controlled failure, and the influence of microstructure can be described in terms of several toughening mechanisms. We provide estimates of the relative importance of these mechanisms.
\end{abstract}


Human bone has a complex hierarchical microstructure ${ }^{6-9}$ that can be considered at many dimensional scales ${ }^{6,7}$. At the shortest length scale, it is composed of type-I collagen fibres (up to $15 \mu \mathrm{m}$ in length, $50-70 \mathrm{~nm}$ in diameter) bound and impregnated with carbonated apatite nanocrystals (tens of $\mathrm{nm}$ in length and width, 2-3 $\mathrm{nm}$ in thickness $)^{6,7}$. These mineralised collagen fibres are further organised at a microstructural length scale into a lamellar structure with roughly orthogonal orientations of adjacent lamellae (3-7 $\mu \mathrm{m}$ thick $)^{8}$. Permeating this lamellar structure are the secondary osteons ${ }^{9}$ (up to 200-300 $\mu$ m diameter): large vascular channels (up to 50$90 \mu \mathrm{m}$ diameter) oriented roughly in the growth direction of the bone, and surrounded by circumferential lamellar rings. The difficulty in understanding the mechanisms of fracture in bone lies in determining the relative importance of these microstructural hierarchies on crack initiation, subsequent crack propagation and consequent unstable fracture and in separating their effects on the critical fracture events.

A vital distinction in the definition of the local (precursor) fracture events that lead to macroscopic failure is whether they are locally stress- or strain-controlled. Brittle fracture, for example in structural steels at very low temperatures, is stresscontrolled, whereas ductile fracture, in the same steels at high temperatures, is straincontrolled (with a significant increase in toughness). Bone fracture is widely regarded as strain-controlled; indeed, most theoretical descriptions of the mechanical behaviour of bone assume this to be the case ${ }^{3}$. However, experimental evidence for this assertion has never been obtained.

To investigate this distinction, we have used a double-notched four-point bend test, which consists of a rectangular bar containing two nominally identical rounded notches (root radius $\sim 200 \mu \mathrm{m}$ ) subjected to four-point bending. The basis of the test is that with a rounded notch in the presence of some degree of inelasticity or yielding ${ }^{10}$, although the maximum local strains are located at the root of the notch ${ }^{2}$, the relaxation of stresses in the inelastic ("yielded") zone surrounding the notch results in the maximum local stresses being located some distance ahead of the notch, close to the 
elastic-inelastic interface ${ }^{11,12}$ (Fig. 1a). (The same is true for a sharp crack although the point of maximum stress is much closer - in the case of shear-driven plasticity, within two crack opening displacements - of the crack tip). Since the two notches experience the same bending moment, when one notch breaks, the other is "frozen" at a point immediately preceding fracture. Examination of the area in the vicinity of the unfractured notch thus reveals the nature of the local fracture event at the onset of failure (area indicated in Fig. 1b "After fracture" illustration).

Since bone displays marked "yielding", Accordingly, we examined the area around several of these unbroken notches in specimens made from a cadaveric human humerus using a traditional scanning electron microscope (SEM). The extremely small $(<5 \mu \mathrm{m})$ size of the precursor cracks that were imaged in all the investigated orientations (Fig. 1c) leaves little doubt that crack initiation is at the notch and not ahead of it (Fig. 2). Moreover, using techniques developed in ref. [12] assuming a plasiticity-based criterion, numerical analysis of the specimens tested at maximum load where the nominal elastic bending stress, $\sigma_{\text {nom }}$, at the notch tip was in the 40-100 MPa range (ratio of nominal stress to yield stress of $\sigma_{\text {nom }} / \sigma_{\mathrm{y}}$ $\sim 0.53-1.33$ ), suggest that the peak tensile stresses should be well ahead of the notch (in fact, at $\sim 100-360 \mu \mathrm{m}$ ahead of the notch tip, i.e., at a distance of roughly 0.5-1.2 times the notch root radius). As absolutely no evidence of any precursor cracking was found in this region and all the initial cracks were detected exactly at the notch root, our observations are consistent with fracture in bone being associated with a strain-based criterion.

Insights into the mechanisms of fracture in bone and how it derives its toughness were also obtained from these experiments. Various toughening mechanisms have been proposed for bone. At large length scales, the generation of "microdamage" from microcracking (small cracks of up to hundreds of micrometers in size) has been suggested as a major source of toughening in bone, specifically via crack-tip shielding ${ }^{13-}$ 15. In addition, the cement lines (at the boundaries of secondary osteons) and the 
interlamellar boundaries are believed to provide weak interfaces that deflect the crack path and accordingly increase the toughness ${ }^{16}$. More recently, a role of collagen fibrils has been postulated ${ }^{17-19}$, with fiber bridging proposed as a possible toughening mechanism ${ }^{20}$. Indeed, toughening at the fibrillar level would explain much of the apparent correlation of toughness with collagen denaturation, which appears to weaken bone, and cross-links, which appear to increase its toughness ${ }^{21}$, although it is probable that many of these mechanisms operate in concert.

Scanning electron micrographs of the fracture paths in human bone, specifically indicating how the crack interacts with the microstructure, are shown in Fig. 3. Fig. 3a shows a roughly $1 \mathrm{~mm}$ long crack propagating out of the notch in the "anti-plane longitudinal" orientation, i.e., the plane of the crack and the crack front are nominally parallel to the long axis of the osteons. It is apparent that at this scale of observation, the most recognizable features of the microstructure, the Haversian canals with their concentric lamellar rings, do not have a major influence on the path taken by the growing crack. Investigation of the near-tip region of this crack, however, shown by the white circle, revealed evidence of so-called uncracked-ligament bridging, as indicated by the white arrow in Fig. 3b. This is an extrinsic toughening mechanism involving two-dimensional uncracked regions along the crack path that can bridge the crack on opening; it is commonly seen in metal-matrix composites ${ }^{22}$ and intermetallics such as $\gamma$ based $\mathrm{TiAl}^{23}$. Such uncracked ligament bridging, however, is more prominent in the "in-plane longitudinal" orientation, as shown by the white arrow in Fig. 2b, where evidence of microcracking can also be seen in the vicinity of the crack. This can also lead to extrinsic toughening through its effect in creating dilation and reducing the modulus in the region surrounding the crack. For the "anti-plane longitudinal" orientation, a third mechanism of toughening in bone can be seen in Fig. $3 \mathbf{c}$ in the form of crack bridging by the collagen fibrils (Fig. 3c).

However, for the "transverse" specimen orientations, where the osteons run along the specimen length (Fig. 1c), we observed a much stronger influence of the underlying 
microstructure on the crack path. Crack initiation and initial crack growth out of the notch was not in the direction normal to the maximum tensile stress, but rather in the direction of the osteons (Fig. 2d). As suggested by Yeni et $a l^{16}$, it would appear that the osteonal cement lines, that are the interface between the osteonal system and the surrounding matrix, provide a weak path for the propagation of the crack, as shown in Fig. 3d. Such large out-of-plane deflections of the crack caused by such behavior leads to substantial toughening (as estimated below) and can be assessed as one of the leading factors associated with the marked anisotropy in the fracture properties of cortical bone.

Such notions on the mechanisms of toughening in bone and how they vary with orientation are consistent with our fracture toughness measurements. Using fatigue precracked bend samples, we measured fracture toughness values of $K_{\mathrm{c}}=5.33( \pm 0.41)$ $\mathrm{MPa} \sqrt{\mathrm{m}}$ for the transverse orientation, as compared to $2.21( \pm 0.18) \mathrm{MPa} \sqrt{\mathrm{m}}$ for antiplane longitudinal orientation and $3.53( \pm 0.13) \mathrm{MPa} \sqrt{\mathrm{m}}$ for the in-plane longitudinal orientation. This is consistent with previous results that give the toughness of bone to be between 2-8 MPa $\sqrt{ } \mathrm{m}^{24-26}$ and to be higher in the transverse direction ${ }^{24,25,27}$.

These observations and measurements can be verified by experiment and theory. The highest toughness is in the transverse orientation where the crack path deflects at 90 degrees to the plane of maxumum tensile stress (Fig. 3d). Linear elastic calculations using crack deflection mechanics ${ }^{28,29}$ suggest that for such an in-plane deviation of the crack path, the stress intensity experienced at the crack tip would be reduced by some $50 \%$ compared to that for an undeflected crack, consistent with the toughness being approximately twice as high in this orientation. A smaller toughening effect is seen in the in-plane longitudinal orientation where crack bridging by uncracked ligaments (and collagen fibrils) is apparent (Fig. 3b). To verify whether such bridging is effective, we compared measurements of the elastic compliance (inverse stiffness) of the cracked bone to those made where we had subsequently machined out the wake of the crack; we also verified the latter measurements by showing that they were identical to the theoretical compliance for a traction-free crack of the same size $\mathrm{e}^{30}$. Results for the anti- 
plane longitudinal orientation are shown in Fig. 3e and clearly indicate that the crack in the bone has a lower compliance than a traction-free crack of identical length. Such results provide strong evidence that cracks in human bone are indeed bridged.

We can quantify the effect of this on the toughening in bone from the difference between the two curves at maximum load, which gives the additional load sustained at the load-line, $P_{\mathrm{br}} \sim 1.5 \mathrm{~N}$. due to the presence of the bridges. This equates to a bridging stress intensity, $K_{\mathrm{br}}$, and hence a contribution to the toughness, of $\sim 0.5 \mathrm{MPa} \sqrt{\mathrm{m}}$, i.e., $1 / 4$ of $K_{\mathrm{c}}$ for this orientation. In comparison, theoretical estimates of ligament bridging, based on a limiting crack-opening approach ${ }^{22}$, yield values of $K_{\mathrm{br}} \sim 0.3 \mathrm{MPa} \sqrt{\mathrm{m}}$. Such experimental compliance measurements, coupled with the theoretical estimates, strongly suggest that uncracked ligament bridging provides a finite contribution to the toughening of bone.

Thus in summary, we have shown by novel experimentation that the local criterion for fracture in human cortical bone is consistent with a strain-based criterion. We believe that this is the first direct experimental evidence for the validity of the assumption of a strain-based criterion, which has been so widely used in theoretical models of the mechanical behavior of bone ${ }^{3}$. In addition, we have shown that the marked anisotropy of bone with respect to its toughness properties can be rationalized in terms of several extrinsic toughening mechanisms induced by specific features in the microstructure. Whereas several of these mechanisms have been proposed, we provided the first quantitative measurements of the contribution to toughening from crack bridging through compliance analysis. These results are of special interest in that they form the basis of physically-based micromechanistic understanding of the fracture and failure of human cortical bone. 


\section{Methods.}

Double notch experiments. A fresh frozen human cadaveric humerus from a 34-year old female was used; the cause of death was unrelated to the condition of the extracted bone. Tests were conducted using a symmetric four-point bending geometry (inner loading span $S \sim 10-15 \mathrm{~mm}$, sample width $W \sim 2-3 \mathrm{~mm}$ ) with a double rounded-notched configuration (Fig. 1b). Rounded notches (root radius, $\rho \sim 200-300 \mu \mathrm{m}$, and depth, $a \sim 0.3-0.4 \mathrm{~W}$ ) were introduced with a slow speed saw; care was taken to maintain the specimens in a hydrated state throughout the specimen preparation and testing process. The depths of both notches in each specimen were kept as identical as possible in order to ensure similar stress/strain fields at the notch tips. A total of eighteen such tests were conducted (atleast three for each of the orientations used- see Fig. 1c). All testing was conducted at ambient temperature on an $\mathrm{ELF}^{\circledR} 3200$ series voice coil-based mechanical testing machine (EnduraTEC Inc., Minnetonka, MN, USA). The bend bars were loaded to failure under displacement control at a constant cross-head movement rate of 0.01 $\mathrm{mm} / \mathrm{sec}$. The area around the unfractured notch (Fig. 1b, "After fracture") was examined using a high-power optical microscope and (after coating with a goldpalladium alloy) with a scanning electron microscope (SEM) operating in the backscattered electron mode.

Fracture toughness measurements. Fracture toughness testing was performed in general accordance with the ASTM Standard E-399 for Plane-Strain Fracture Toughness $^{31}$. Tests were conducted using the notched three-point bending geometry with a span between the lower two loading points equal to 5-5.5 times the width of the beam. Both the longitudinal orientations and the transverse orientation were investigated. The notch was "sharpened" by "precracking" using cyclic fatigue loading; this was achieved at a load ratio (ratio of minimum to maximum loads) of $R=0.1$ and loading frequency of $2 \mathrm{~Hz}$, with a final maximum stress-intensity of $K_{\max } \sim 1-2 \mathrm{MPa} \sqrt{\mathrm{m}}$. The final precrack length (notch plus precrack) was generally $\sim 0.4-0.6 \mathrm{~W}$, with a presumed atomically sharp crack tip. Samples were then loaded to failure under 
displacement control with an $\mathrm{ELF}^{\circledR} 3200$ series machine at ambient temperature at a cross-head displacement rate of $0.01 \mathrm{~mm} / \mathrm{sec}$. A record of the applied loads and the corresponding displacements was simultaneously monitored during the test and analyzed for determining the fracture toughness. At least three separate specimens were tested for each orientation. Linear-elastic stress intensities, $K$, were computed from handbook solutions for three-point bending ${ }^{31}$.

Crack bridging experiments. To confirm the presence of crack bridging in bone, several experiments $(N=4)$ were performed where a crack was grown from a rounded notch in a three-point bend specimen to a certain length between 1 and $1.5 \mathrm{~mm}$. The "measured" compliance of this crack was then determined by monitoring the load-line displacement as a function of applied bending load. This was then compared to the "theoretical" compliance for a traction-free crack of identical length in this geometry ${ }^{32}$. To check the veracity of the theoretical estimate for the case of bone, we machined out the wake of the crack using a slow speed diamond saw to obtain a nominally tractionfree "crack" of the same length, and measured its compliance (see Fig. 3e).

\section{References}

1. Ritchie, R. O., Knott, J. F. \& Rice, J. R. On the relationship between critical tensile stress and fracture toughness in mild steel. J. Mech. Phys. Solids 21, 395-410 (1973).

2. Ritchie, R. O., Server, W. L. \& Wullaert, R. A. Critical fracture stress and fracture strain models for the prediction of lower and upper shelf toughness in nuclear pressure vessel steels. Metall. Trans. A 10A, 1557-1570 (1979).

3. Yeh, O. C. \& Keaveny, T. M. Relative roles of microdamage and microfracture in the mechanical behavior of trabecular bone. J. Orthopaed. Res. 19, 1001-1007 (2001). 
4. Keyak, J. H. \& Rossi, S. A. Prediction of femoral fracture load using finite element models: an examination of stress- and strain-based failure theories. J. Biomech. 33, 209$214(2000)$.

5. Lewandowski J. J. \& Thompson A. W. Microstructural effects on the cleavage fracture stress in fully pearlitic 1080 steel. Advances in Fracture Research (Fracture 84): Proc. $6^{\text {th }}$ Int. Conf. on 'Fracture', Pergamon Press, New York, NY, USA, 1515$1522(1984)$.

6. http://ttb.eng.wayne.edu/ grimm/ME518/L3F3.html (From Park, J.B. \& Lakes, R.S. Biomaterials: An Introduction (Plenum Press, New York, NY, USA, 1992)).

7. Rho, J.Y., Kuhn-Spearing, L. \& Zioupos, P. Mechanical properties and the hierarchical structure of bone. Med. Eng. Phys. 20, 92-102 (1998).

8. The finer details of the collagen orientation within a lamella are still an area of active investigation (e.g., Weiner, S. \& Wagner, H.D. Ann. Rev. Mater. Sci. 28, 271-298 (1998).

9. Currey, J.D. 'Osteons' in biomechanical literature. J. Biomech. 15, 717 (1982).

10. It is appreciated that "yielding" in bone cannot be simply related to shear-driven plasticity, e.g., in metals, for which the notch-field solutions in Fig. 1a were explicitly derived. Indeed, the precise nature of the inelastic constitutive behavior of bone is not known, but clearly involves diffuse microcracking damage and plasticity in the collagen fibrils, which would be sensitive to both tensile and shear stresses (somewhat akin to pressure-dependent yielding in polymers). Despite this, most theoretical models for both deformation (e.g., Zioupos, P. et al., Philos. Trans. R. Soc. Lond. B. Biol. Sci. 347, 383-396 (1995)) and fracture (e.g., Lotz, J. C., Cheal, E. J. \& Hayes, W. C. J. Biomech. Eng. 113, 353-360 (1991)) in bone simply utilize the von Mises criterion, which is based on pressure-insensitive plasticity theory. It is in this spirit that we use the notch- 
field solutions in Fig. 1a, but we recognize that the quantitative details of these fields have yet to be determined for realistic constitutive relationships in bone.

11. Hill, R. The Mathematical Theory of Plasticity (Clarendon Press, Oxford, U.K., 1950).

12. Griffiths, J.R. \& Owen, D. R. J. An elastic-plastic stress analysis for a notched bar in plane strain bending. J. Mech. Phys. Solids 19, 419-431 (1971).

13. Vashishth, D. et al. In vivo diffuse damage in human vertebral trabecular bone. Bone 26, 147-152 (2000).

14. Parsamian G. P. \& Norman, T. L. Diffuse damage accumulation in the fracture process zone of human cortical bone specimens and its influence on fracture toughness, J. Mater. Sci.: Mater. Med. 12, 779-783 (2001).

15. Vashishth, D., Tanner, K. E. \& Bonfield, W. Contribution, development and morphology of microcracking in cortical bone during crack propagation J. Biomech. 33, 1169-1174 (2000).

16. Yeni, Y. N. \& Norman, T. L. Calculation of porosity and osteonal cement line effects on the effective fracture toughness of cortical bone in longitudinal crack growth. J. Biomed. Mater. Res. 51, 504-509 (2000).

17. Wang, X., Bank, R. A., Tekoppele, J. M. \& Agrawal, C. M. The role of collagen in determining bone mechanical properties. J. Orthopaed. Res. 19, 1021-1026 (2001).

18. Wang, X., Shen, X., Li, X. \& Agrawal, C. M. Age-related changes in the collagen network and the toughness of bone. Bone 31, 1-7 (2002).

19. Thompson, J.B. et al. Bone indentation recovery time correlates with bond reforming time. Nature 414, 773-776 (2001). 
20. Yeni, Y. N. \& Fyhrie, D. P. Collagen-bridged microcrack model for cortical bone tensile strength. Proc Bioengineering Conference BED 50, ASME, New York, NY, USA, 293-294 (2001).

21. Burr, D. B. The contribution of the organic matrix to bone's material properties. Bone 31, 8-11 (2002).

22. Shang, J. H. \& Ritchie, R. O. Crack bridging by uncracked ligaments during fatiguecrack growth in SiC-reinforced aluminum-alloy composites. Metall. Trans. A 20A, 897908 (1989).

23. Campbell, J. P., Venkateswara Rao, K. T. \& Ritchie, R. O. The effect of microstructure on fracture toughness and fatigue crack growth behaviour in $\gamma$-titanium aluminide based intermetallics. Metall. Mater. Trans. A 30A, 563-577 (1999).

24. Phelps, J. B., Hubbard, G. B., Wang, X. \& Agrawal, C. M. Microstructural heterogeneity and the fracture toughness of bone. J. Biomed. Mater. Res. 51, 735-741 (2000).

25. Lucksanambool, P., Higgs, W. A. J., Higgs, R. J. E. D. \& Swain, M. W. Fracture toughness of bovine bone: influence of orientation and storage media. Biomater. 22, 3127-3132 (2001).

26. Zioupos, P. \& Currey, J. D. Changes in the stiffness, strength, and toughness of human cortical bone with age. Bone 22, 57-66 (1998).

27. Behiri, J. C. \& Bonfield, W. Orientation dependence on fracture mechanics of bone. J. Biomech. 22, 863-872 (1989).

28. Bilby, B. A., Cardew, G. E. \& Howard, I. C. Stress intensity factors at the tips of kinked and forked cracks. Fracture 1977 3, Pergamon Press, Oxford, UK, 197-200 (1978). 
29. Cotterell, B. \& Rice, J. R. Slightly curved or kinked cracks. Int. J. Fract. 16, 155$169(1980)$.

30. Ritchie, R. O., Yu, W. \& Bucci, R. J. Fatigue crack propagation in arall laminates: measurement of the effect of crack-tip shielding from crack bridging. Eng. Fract. Mech. 32, 361-377 (1989).

31. ASTM E 399-90 (Reapproved 1997). Annual Book of ASTM Standards, Vol. 03.01: Metals- Mechanical testing; Elevated and low-temperature tests; Metallography (ASTM, West Conshohocken, PA, USA, 2001).

32. Haggag, F. M. \& Underwood, J. H. Compliance of a three-point bend specimen at load line. Int. J. Fract. 26, R63-R65 (1984).

\section{Acknowledgments}

This work was supported by the National Institutes of Health under Grant No. P01DE09859 (for RKN) and by the Office of Science, Office of Basic Energy Science of the Department of Energy under Contract No. DE-Ac03-76SF00098 (for ROR). The authors wish to thank Christian M. Puttlitz for supplying the human bone used in this study, Dr. Jamie J. Kruzic for assistance with the compliance measurements, and Prof. John W. Hutchinson for helpful discussion.

\section{Competing interests statement}

The authors declare that they have no competing financial interests. 


\section{Figure Legends:}

Figure 1 Schematics illustrating the difference between failure criteria, the test geometry and the orientations used: a The stress (left) and strain (right) distributions, based on numerical computations ${ }^{12}$, ahead of a notch are shown, and indicate that, in the presence of any degree of inelasticity, the maximum stresses are ahead of the notch whereas the maximum strains are at the notch root. Consequently, a stress-controlled fracture mechanism will tend to initiate ahead of the notch, whereas the initial fracture event for strain-controlled fracture will be at the notch. b Schematic illustration of the double-notched fourpoint bend test used to discern whether fracture is stress- or strain-controlled. c The various specimen orientations taken from the humerus (with respect to the direction of the osteons, indicated in gray) that were investigated are shown in this illustration.

Figure 2 Scanning electron micrographs of the area near the unbroken notch for various orientations: a A crack emanating directly from the notch root in the "anti-plane longitudinal" orientation. b Associated evidence of uncracked ligament bridging (indicated by white arrow) and of extensive microcracking for the "in-plane longitudinal" orientation. c The extremely small $(<5 \mu \mathrm{m})$ size of the precursor cracks like the one shown here leaves little doubt that crack initiation is at the notch and not ahead of it, consistent with the notion of locally straincontrolled fracture. d The strong influence of the underlying microstructure can lead to cracks emanating well behind the notch root, as illustrated here for the "transverse" orientation. Also, multiple crack initiation can be seen. The insets show the specimen orientation with respect to the direction of the osteons. 
Figure 3 Scanning electron micrographs illustrating toughening mechanisms in bone through interactions between the crack and the microstructure interactions: a For a crack emanating from the notch in the "anti-plane longitudinal" orientation, the crack path appears to be little influenced by the osteons (encircled by the white arrows). b A high magnification image of the region indicated in a by a white circle showing evidence of uncracked ligament bridging as a contribution to the toughness (indicated by white arrows). c A high magnification micrograph showing crack bridging by collagen fibrils, also for "anti-plane longitudinal" orientation. d A stronger influence of microstructure is evident for the "transverse" orientation, where cracking ahead of the notch is shown at a Haversian canal, although the actual initiation process is at the notch itself, as evidenced by the presence of precursor cracks (Fig. 2d). The insets show the specimen orientation with respect to the direction of the osteons; the black arrows indicate the nominal direction of crack growth. $\mathbf{e}$ Experimental (bridged) and theoretical (traction-free) load-displacement curves used to assess the specimen compliance to verify the existence (and quantify) the bridging levels involved (at constant crack length). 


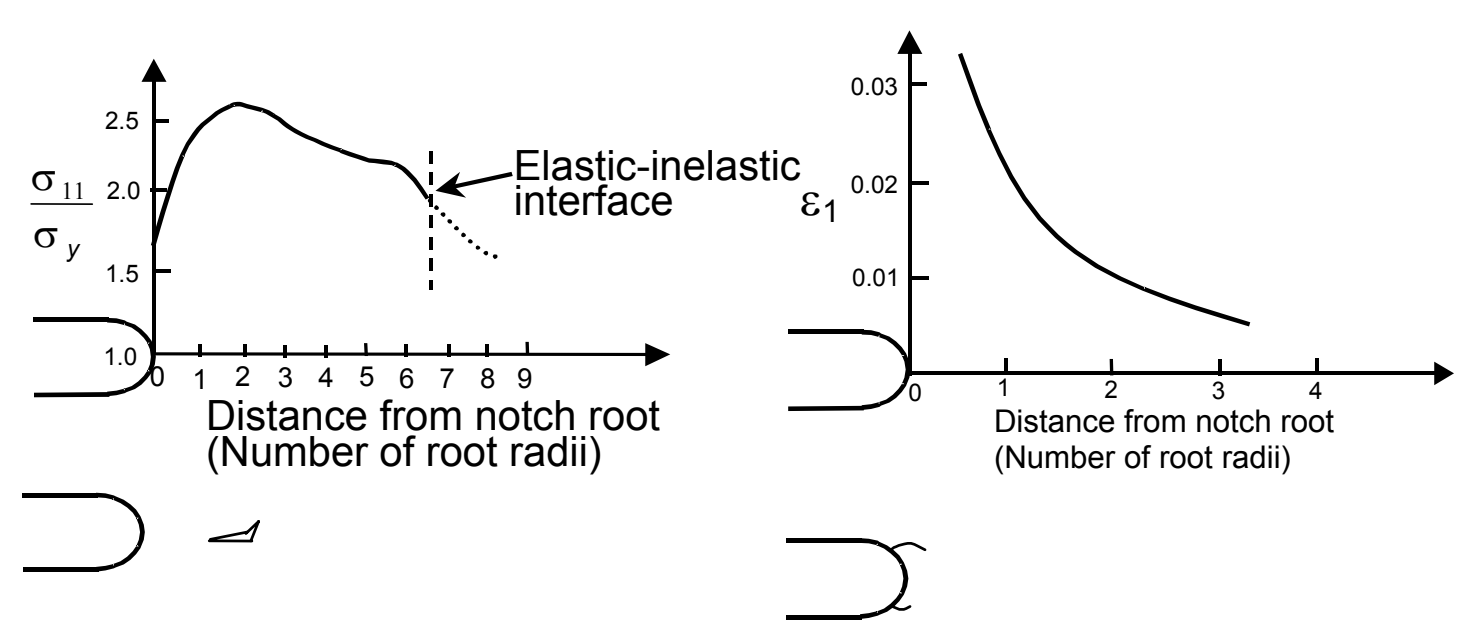

Stress-controlled fracture

a
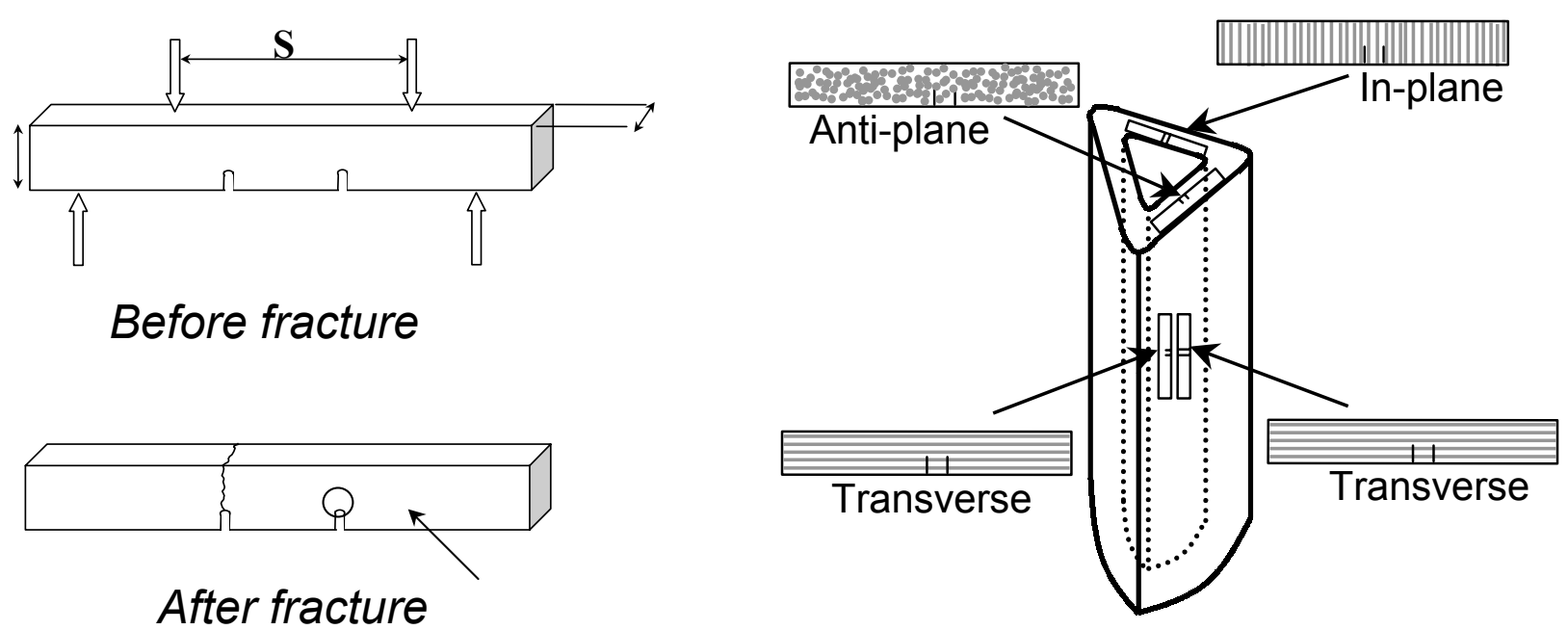

b

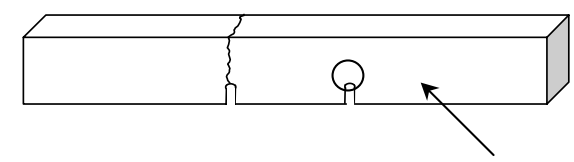

After fracture
Strain-controlled fracture

C

Figure 1 Schematics illustrating the difference between failure criteria, the test geometry and the orientations used: a The stress (left) and strain (right) distributions, based on numerical computations ${ }^{12}$, ahead of a notch are shown, and indicate that, in the presence of any degree of inelasticity, the maximum stresses are ahead of the notch whereas the maximum strains are at the notch root. Consequently, a stress-controlled fracture mechanism will tend to initiate ahead of the notch, whereas the initial fracture event for strain-controlled fracture will be at the notch. b Schematic illustration of the double-notched fourpoint bend test used to discern whether fracture is stress- or strain-controlled. c The various specimen orientations taken from the humerus (with respect to the direction of the osteons, indicated in gray) that were investigated are shown in this illustration. 

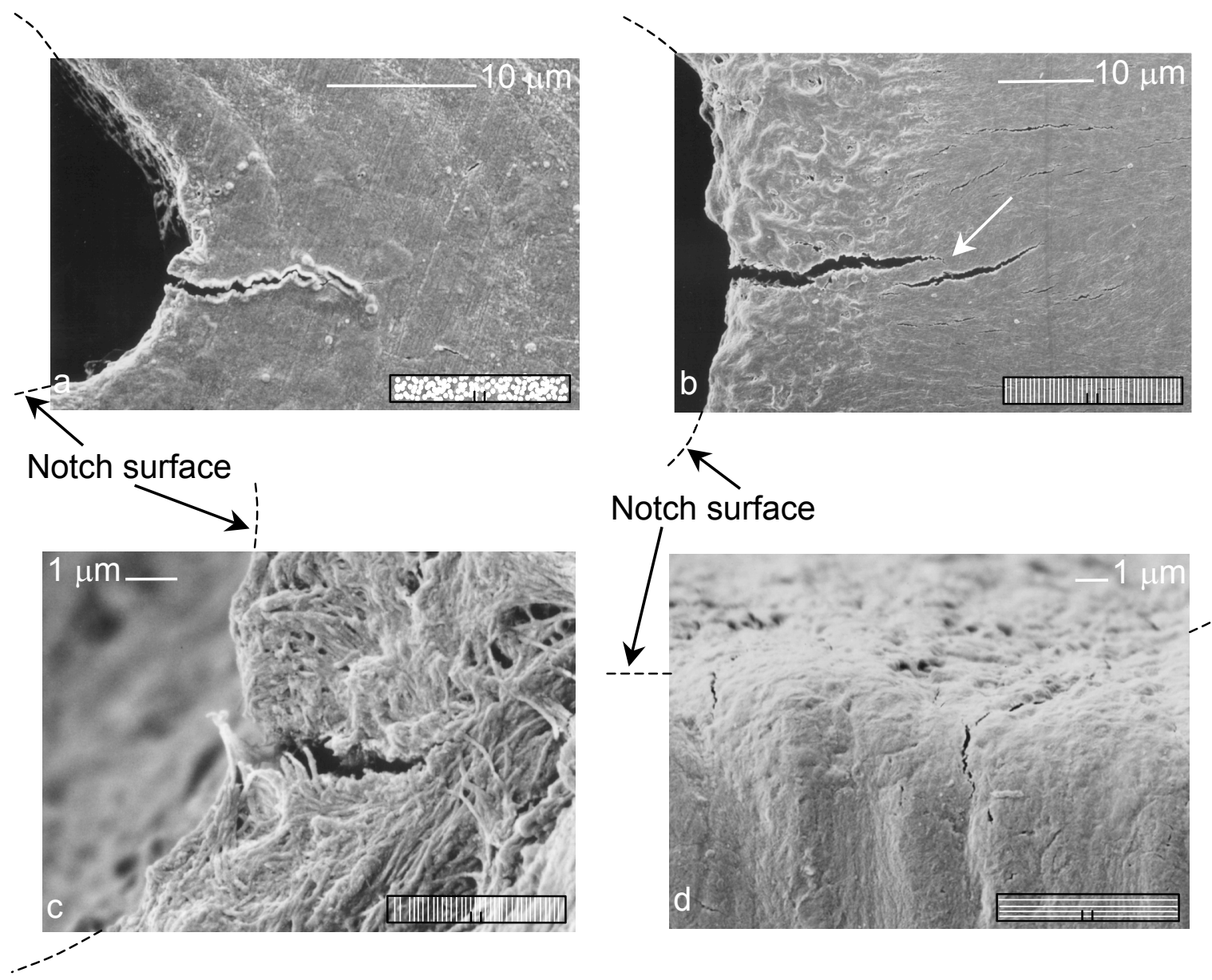

Figure 2 Scanning electron micrographs of the area near the unbroken notch for various orientations: a A crack emanating directly from the notch root in the "anti-plane longitudinal" orientation. b Associated evidence of uncracked ligament bridging (indicated by white arrow) and of extensive microcracking for the "in-plane longitudinal" orientation. c The extremely small $(<5 \mu \mathrm{m})$ size of the precursor cracks like the one shown here leaves little doubt that crack initiation is at the notch and not ahead of it, consistent with the notion of locally straincontrolled fracture. d The strong influence of the underlying microstructure can lead to cracks emanating well behind the notch root, as illustrated here for the "transverse" orientation. Also, multiple crack initiation can be seen. The insets show the specimen orientation with respect to the direction of the osteons. 

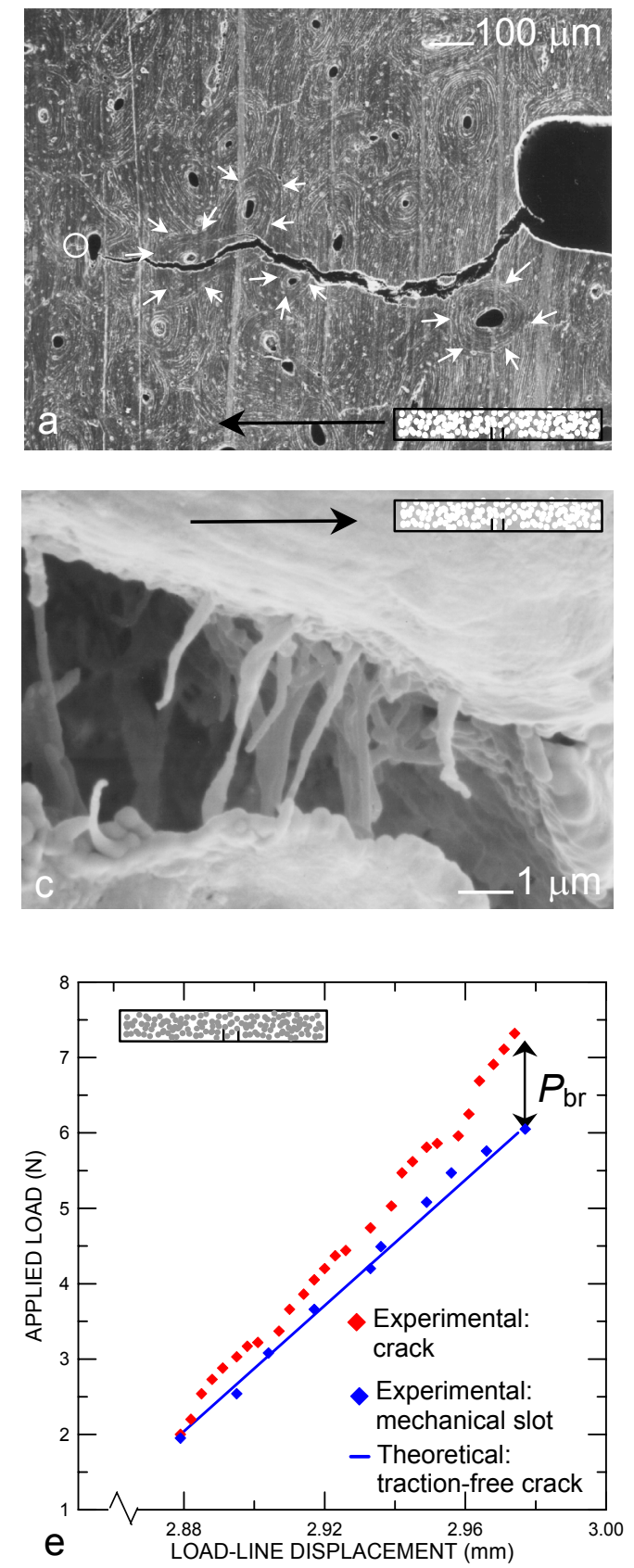

Figure 3 Scanning electron micrographs illustrating toughening mechanisms in bone through interactions between the crack and the microstructure interactions: a For a crack emanating from the notch in the "anti-plane longitudinal" orientation, the crack path appears to be little influenced by the osteons (encircled by the white arrows). b A high magnification image of the region indicated in a by a white circle showing evidence of uncracked ligament bridging as a contribution to the toughness (indicated by white arrows). c A high magnification micrograph showing crack bridging by collagen fibrils, also for "anti-plane longitudinal" orientation. d A stronger influence of microstructure is evident for the "transverse" orientation, where cracking ahead of the notch is shown at a haversian canal, although the actual initiation process is at the notch itself, as evidenced by the presence of precursor cracks (Fig. 2d). The insets show the specimen orientation with respect to the direction of the osteons; the black arrows indicate the nominal direction of crack growth. e Experimental (bridged) and theoretical (traction-free) load-displacement curves used to assess the specimen compliance to verify the existence (and quantify) the bridging levels involved (at constant crack length). 

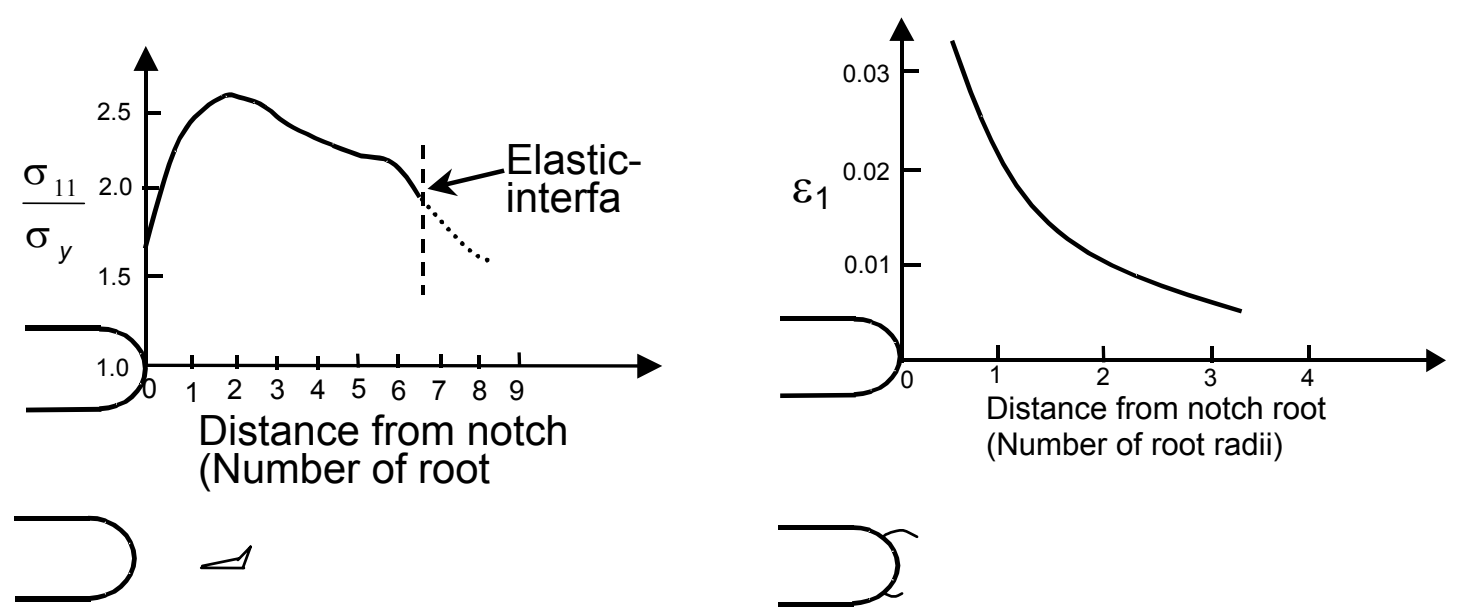

Stress-controlled fracture

Strain-controlled fracture

a
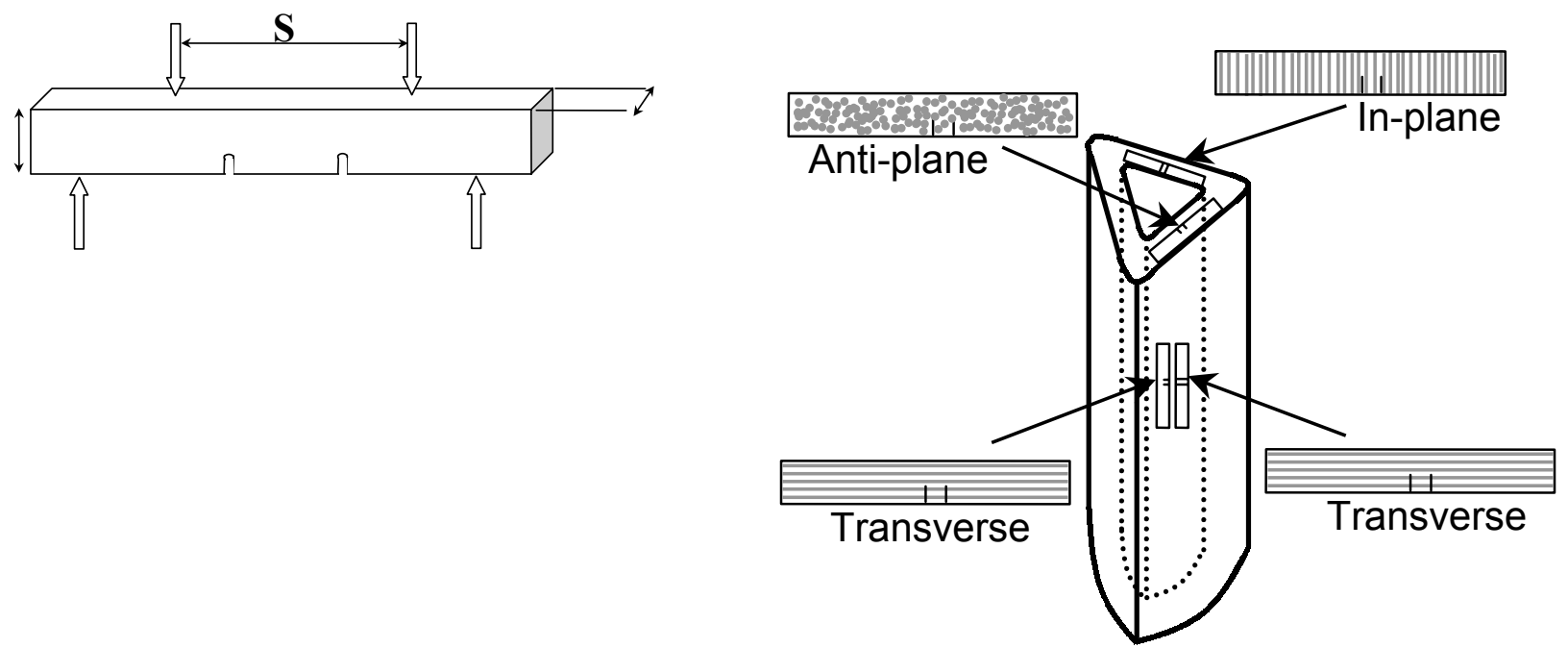


\section{Before fracture}

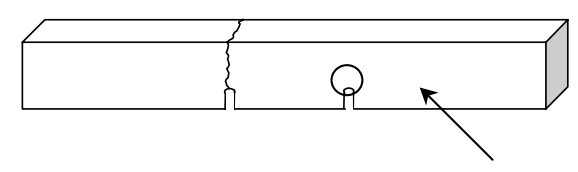

After fracture

b

C 

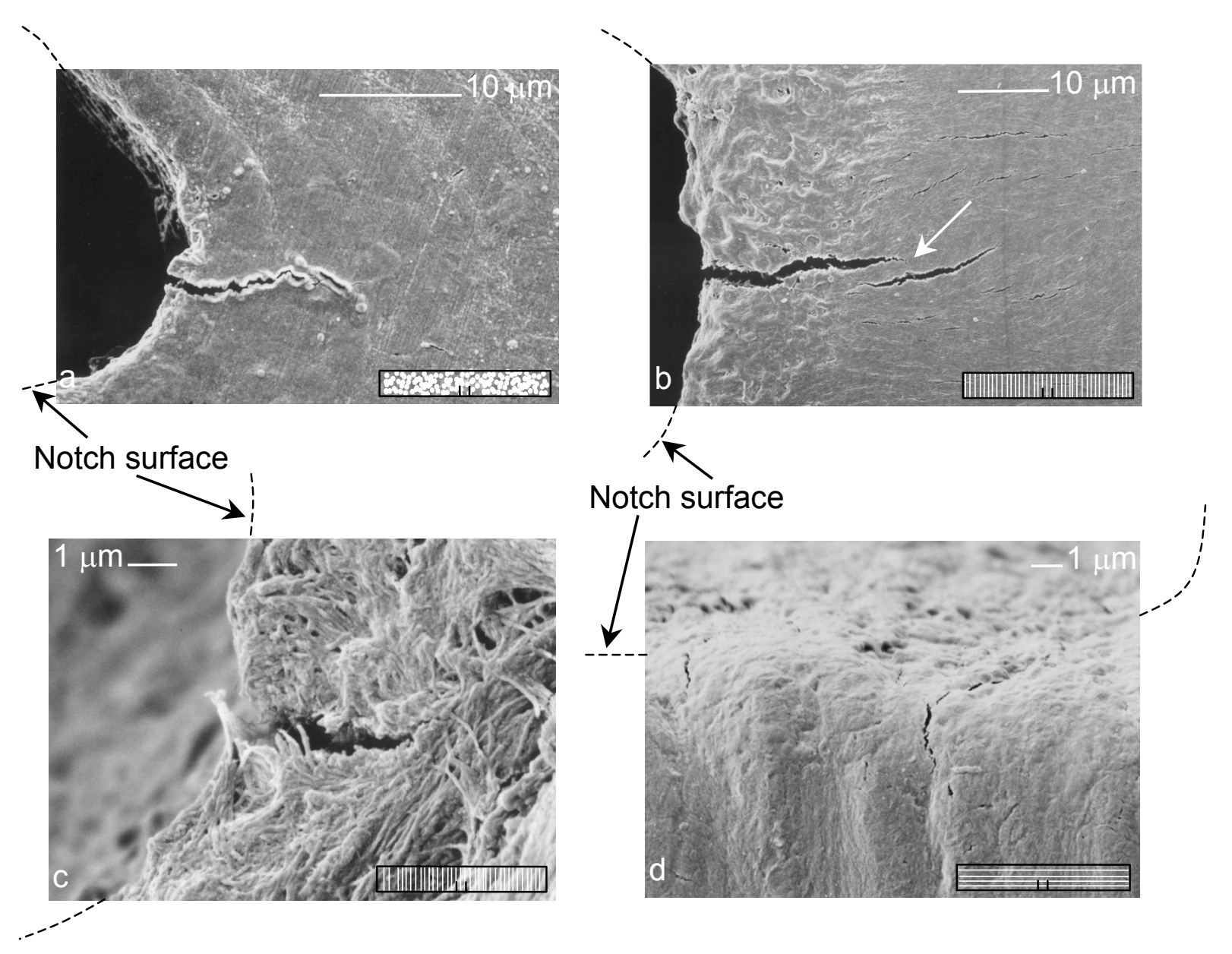

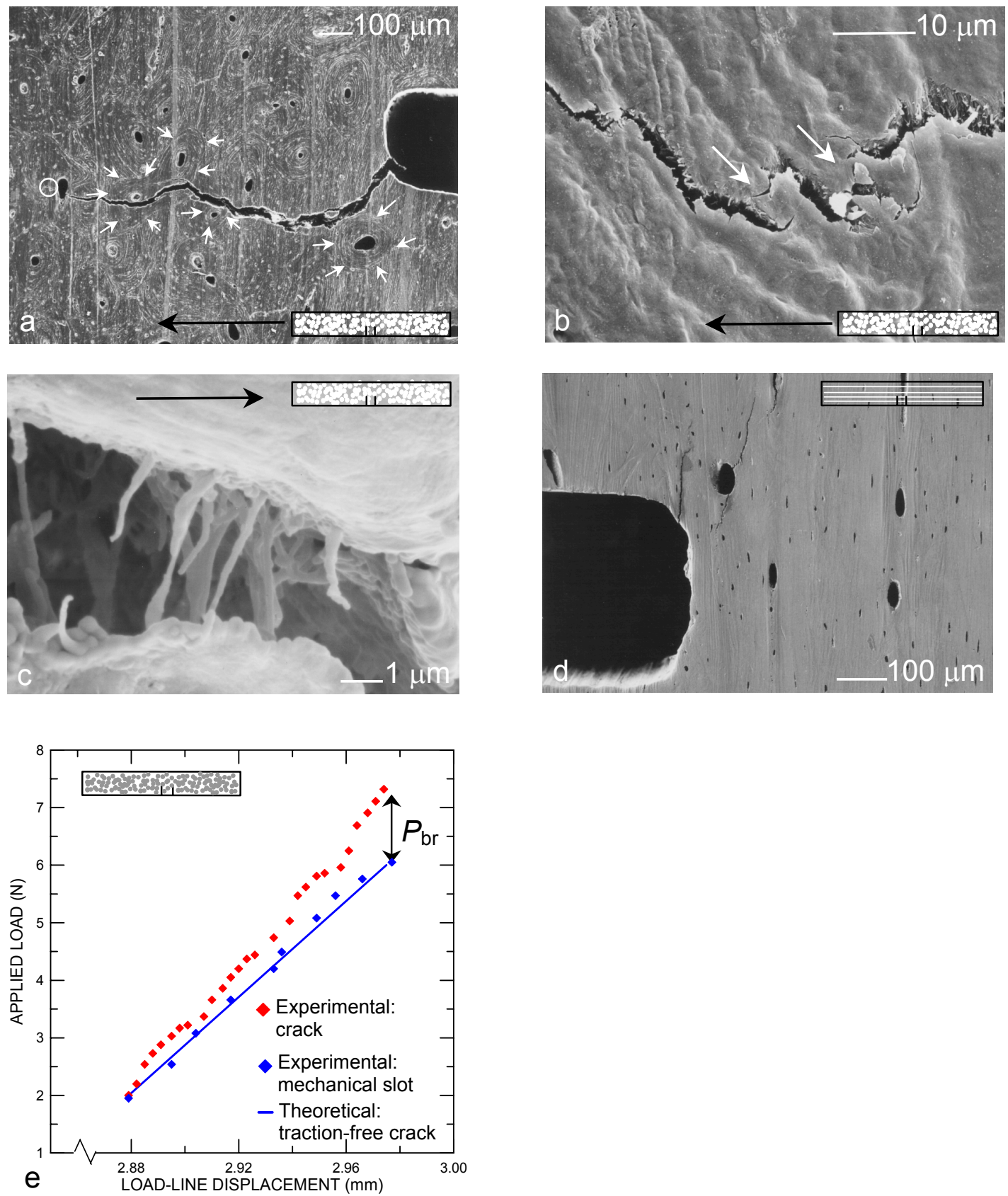